\title{
High - Resolution Ultrasound Imaging for Angioleiomyoma: A Painful and Vascularized Superficial Tumor
}

\author{
Kazumi Fujioka*1, Akira Fujioka ${ }^{2}$, Minoru Oishi ${ }^{3}$, Ken Hayashi ${ }^{4}$ and Tomohiro Nakayama ${ }^{1}$ \\ ${ }^{1}$ Department of Pathology and Microbiology, Nihon University School of Medicine, Japan \\ ${ }^{2}$ Department of Dermatology, Fujioka Dermatological Clinic, Japan \\ ${ }^{3}$ Department of Internal Medicine, Izutobu General Hospital, Japan \\ ${ }^{4}$ Department of Dermatology, Tokyo Rosai Hospital, Japan
}

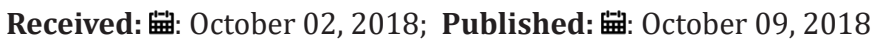

*Corresponding author: Kazumi Fujioka, Division of Laboratory Medicine, Department of pathology and Microbiology, Nihon University School of Medicine, 30-1 Oyaguchi-kamicho, Itabashi-Ku, Japan

\begin{abstract}
Angioleiomyoma (ALM) is a rare benign, vascular smooth muscle tumor originating from the tunica media of the vessel wall. It typically arises in the cutaneous, subcutaneous tissue of the lower extremities in middle-aged women and is less than $2 \mathrm{~cm}$ in diameter. We report an ALM of the thigh in a 69-year-old woman with intermittent pain. US was performed with a high-resolution, broad-band (5 MHz-18 MHz) linear transducer for the superficial nodule. To the best of our knowledge, there has been no report of high-resolution ultrasound image features, including grayscale US, color Doppler US and Real-Time Tissue Elastography (RTE) for an ALM. ALM should be considered as one of the painful and vascularized subcutaneous mass, a superficial location that can be seen on high-resolution US. The feature of adjacent blood flow signal on color Doppler US could be strongly suggested to be ALM.
\end{abstract}

Keywords: High - Resolution Ultrasound; Angioleiomyoma; Adjacent Blood Flow Signal; Painful and Vascularized Superficial Tumor; Color Doppler Ultrasonography

\section{Introduction}

ALM is a rare tumor that arises from the tunica media of small arteries and veins [1]. The tumors are usually small, solitary, round, painful, firm, skin-colored, and well-encapsulated [2]. Dermatologic ultrasound imaging has been rapidly growing in recent years because of the development of high-resolution multifrequency transducers and multichannel color Doppler machines [3]. We report a case of subcutaneous ALM, a superficial location that can be seen on high-resolution US. A high-resolution, broad-band (5 MHz-18 MHz) linear transducer was used for all scans, including gray-scale US, color Doppler US and RTE in our case. On gray-scale US, there are some reports $[4,5]$ that the lesions were described as circumscribed homogeneous hypoechoic solid nodule. There are a few reports [4-6] concerning the imaging features on color Doppler US. Present case showed solid nodule pattern both on gray-scale US and RTE. Vascular flow signals were seen in both peripheral and central on color Doppler US in the present case. The peripheral flow signal on color Doppler US was suggested to be consistent with adjacent vessel. ALM is suspected in the female case of a painful, solid nodule of the extremities. ALM should be considered as one of the painful and vascularized subcutaneous mass, a superficial location that can be seen on high-resolution US. ALM mimic schwannoma very closely, appearing as well-defined hypoechoic, round nodules with internal vascularity. The detection of adjacent blood flow signal is a useful appearance in relation to the differentiation ALM from schwannoma on color Doppler US.

\section{Case Report}

A 69-year-old female, having noticed a nodule in her lower extremity, visited a nearby dermatologist in February 2014. The nodule was firm and slightly tender to touch. The patient noticed the nodule 20 years earlier. The nodule was small, solitary, round, painful and firm. US was performed with a high-resolution, broadband (5 MHz-18 MHz) linear transducer (Nobulus Hitachi, Ltd. Tokyo, Japan). Gray scale US showed an oval shaped, circumscribed, homogeneous, hypoechoic lesion. The lesion is in a superficial location, possibly in close to the dermis in $4 \mathrm{~mm}$ depth. The size of the nodule is $2 \mathrm{~mm}$ in depth and $3 \mathrm{~mm}$ in width. Posterior echo enhancement was absent on gray-scale US, suggesting the solid tumor pattern. (Figure 1a). Central and peripheral blood 
flow signals were detected on color Doppler US (Figure 1b). Spectral Doppler analysis demonstrated low blood flow velocity within the nodule (Figure 2a). On RTE, the nodule was depicted as mostly blue color, consistent with the hard nature mass [7] (Figure 2b). Operation was performed due to the painful nodule. Histopathological finding of the lesion showed proliferation of the blood vessels and intervascular smooth muscle bundles (Figure 3a). Nodule was pathologically diagnosed as ALM (Figure 3b).

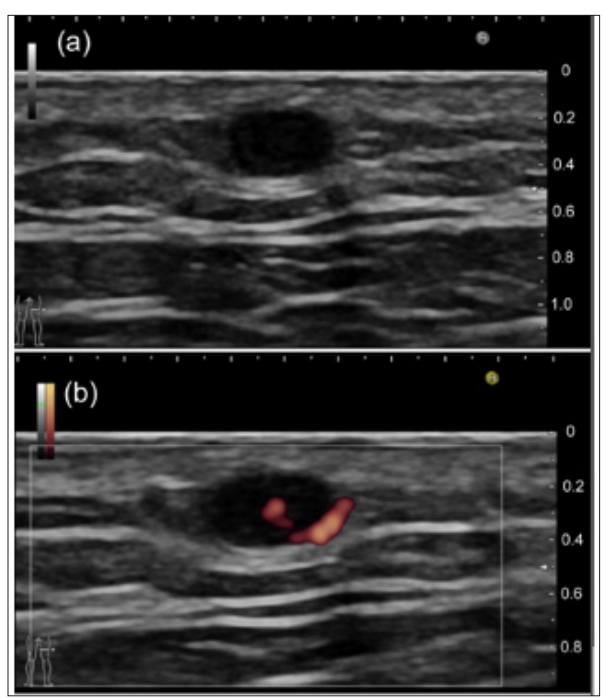

Figure 1: (a) Gray-scale US shows an oval shaped, circumscribed, homogeneous, hypoechoic nodule without posterior echo enhancement. The lesion is in a superficial location in $4 \mathrm{~mm}$ depth. (b) Central and peripheral blood flow signals were detected on color Doppler US. Peripheral flow signal on color Doppler US was suggested to be consistent with adjacent vessel.
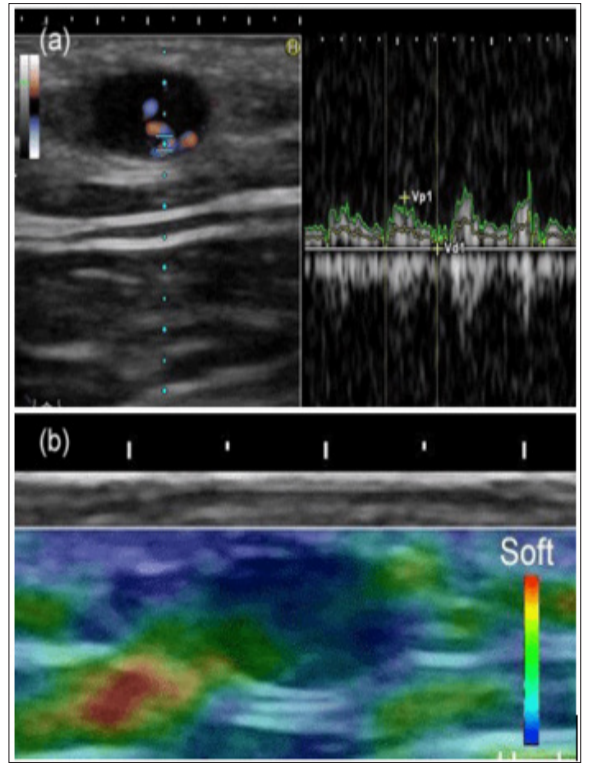

Figure 2: (a) Spectral Doppler analysis demonstrated low blood flow velocity within the nodule. (b) On Real-time tissue elastography, the nodule was depicted as mostly blue color, consistent with the hard nature mass.

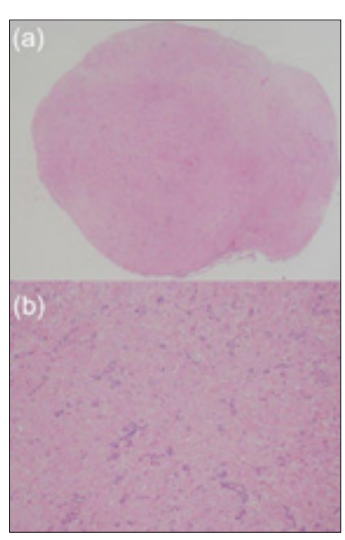

Figure 3: (a) Histopathological finding of the lesion showed proliferation of the blood vessels and intervascular smooth muscle bundles. (hematoxylin and eosin, original magnification X20). (b) The nodule was pathologically diagnosed as ALM. (hematoxylin and eosin, original magnification X100).

\section{Discussion}

ALM is a rare tumor that arises from the tunica media of small arteries and veins [1]. The tumors are usually small, solitary, round, painful, firm, skin-colored, and well-encapsulated [2]. Dermatologic ultrasound imaging has been rapidly growing in recent years because of the development of high-resolution multifrequency transducers and multichannel color Doppler machines [3]. Highresolution ultrasound, at $5-18 \mathrm{MHz}$, have been used to image the skin and subcutaneous lesions. Raffin D et al. reported highfrequency ultrasound (HFU) imaging with 25-MHz linear probe for cutaneous neurofibroma in patients with neurofibromatosis type 1. They showed HFU of dermal lesion located in 4-6 mm depth [8]. Mandava A et al. reported that normal skin is made of three layers: the epidermis (thickness $0.06-0.6 \mathrm{~mm}$ ) on the most external surface; the underlying dermis (thickness 1-4 mm) consisting of connective tissue, nerves, blood, and lymphatic vessels, gland, mast cells, fibroblasts, histiocytes, etc.; and the subcutaneous tissue (thickness 5-20 mm), mainly made of adipose cells [9]. We report a case of subcutaneous ALM, a superficial location, probably in close to the dermis located in $5 \mathrm{~mm}$ depth on high-resolution US.

A high-resolution, broad-band (5 MHz-18 MHz) linear transducer was used for all scans, including gray-scale US, color Doppler US and RTE. The nodule was depicted as hard nature with low elasticity on RTE [7], which was consistent with the solid tumor pattern without posterior echo enhancement on gray-scale US. Image features showed solid tumor pattern both on gray-scale US and RTE. The type of the nerve-tumor position and transition are valuable feature in neurogenic tumor [10]. This appearance of nerve-tumor connection was not detected in our case. Blood flow signals were detected in central and peripheral on color Doppler US, suggesting type 1 pattern as described previously [5]. Peripheral blood flow signal was suggested to be adjacent vessel as previously described [11]. Concerning the imaging features on gray scale and color Doppler US, some studies have been reported [4-6]. Zhang JZ et al. described typical sonographic features of ALM as subcutaneous mass, 
a superficial location, especially in close to or contact with the dermis, a small size (20 mm), an oval shape, complete solid components, homogeneous hypoechogenicity, low or moderate vascular density [4]. Park HJ et al. [5] reported that AML showed a homogeneous hypoechoic echotexture with a circumscribed margin and straight and linear vessels in the tumors with convergence to one point on color Doppler US [5].

Several tumors containing ALM, eccrine spiradenoma, neuroma, dermatofibroma, angiolipoma, schwannoma, glomus tumor, have been clinically known such as a painful nodule. It has been suggested that pain may be due to local tissue anoxia or to compression of cutaneous nerves by the tumor [1]. Meanwhile, vascularized superficial benign soft-tissue tumors are ultrasonographically regarded as eccrine spiradenoma, dermatofibroma, schwannoma, vascular leiomyoma, glomus tumor, pilomatricoma, chondroid syringoma, hemangioma [12]. Differential diagnosis are as follows: Eccrine spiradenoma are rare benign tumors of the sweat gland, showing hererogeneously hypoechoic with lobular margin on grayscale US and with increased peripheral portion, with or without blood flow in the central portion on color Doppler US [13]. Diffusely scattered irregular vessels are depicted on color Doppler US and spectral analysis demonstrated high speed and high resistance profile in nonsubungual glomus tumors [14]. Dermatofibroma shows a well-defined margin and hypoechogenicity in the dermisto-subcutaneous fat layer with increased vascular flow [12]. Focal vascular flows are noted in hemangioma. On compression with transducer, more increased vascular flow is depicted in the mass.

ALM shows variable vascular pattern, density and distribution as previously described [5]. The nerve-tumor association and vascularity of the tumors could be useful for differentiation of between schwannoma and neuroma. Schwannoma shows eccentric nerve-tumor position on gray-scale US and vascularity within the tumor on color Doppler US [10]. ALM mimic schwannoma very closely, appearing as well-defined hypoechoic, round nodules with internal vascularity; however, they show peripheral blood flow signal on color Doppler US, which is consistent with adjacent vessel, rather they do not show eccentric nerve-tumor position on grayscale US. The features of nerve-tumor association and adjacent vessel could be useful for differentiation of between schwannoma and ALM. A High-resolution, broad-band (5 MHz-18 MHz) linear transducer was used for all scans, including gray-scale US, color Doppler US and RTE in our case. ALM is suspected in the female case of a painful, solid nodule of the extremities. We suggest that most of painful nodule show vascularity within the tumor. ALM should be considered as one of the painful and vascularized subcutaneous mass, a superficial location, possibly in close to the dermis, that can be seen on high-resolution color Doppler US.

\section{Conclusion}

Angioleiomyoma should be considered as one of the painful and vascularized superficial soft tissue tumor that can be depicted on high-resolution color Doppler US. The feature of adjacent blood flow signal on color Doppler US could be strongly suggested to be angioleiomyoma.

\section{References}

1. Tejasvi BR, Pai K, Rao R (2014) Painful nodule on the leg. Clin Exp Dermatol 39(4): 542-543.

2. Chen YA, Chuang WY, Hsuceh S, Chan KC (2012) Solitary nodule on the auricle. Int J Dermatol 51(12): 1427-1428.

3. Wortsman X, Alfageme F, Roustan G, Arias-Santiago S, Martorell A, et al. (2016) Guidelines for performing dermatologic ultrasound examinations by the DERMUS group. J Ultrasound Med 35(3): 577-580.

4. Zhang JZ, Zhou J, Zhang ZC (2016) Subcutaneous angioleiomyoma: clinical and sonographic features with histopathologic correlation. J Ultrasound Med 35(8): 1669-1673.

5. Park HJ, Kim SS, Lee SY, Choi YJ, Chung EC, et al. (2012) Sonographic appearances of soft tissue angioleiomyomas: differences from other circumscribed soft tissue hypervascular tumors. J Ultrasound Med 31(10): 1589-1595.

6. Oiso N, Narita T, Kawada A (2013) Diagnostic usefulness of ultrasonography for plantar angioleiomyoma. Eur J Dermator 23(4): 568-570.

7. Nakashima K, Shiina T, Sakurai M, Enokido K, Endo T, et al. (2013) JSUM ultrasound elastography practice guidelines: breast. J Med Ultrason 40(4): 359-391.

8. Raffin D, Zaragoza J, Georgescou G, Mourtada Y, Maruani A, et al. (2017) High-frequency ultrasound imaging for cutaneous neurofibroma in patients with neurofibromatosis type 1. Eur J Dermator 27(3): 260-265.

9. Mandava A, Ravuri PR, Konathan R (2013) High-resolution ultrasound imaging of cutaneous lesions. Indian J Radiol Imaging 23(3): 269-277.

10. Ryu JA, Lee SH, Cha EY, Kim TY, Kim SM, et al. (2015) Sonographic differentiation between schwannomas and neurofibromas in the musculoskeletal system. J Ultrasound Med 34(12): 2253-2260.

11. Yoo HJ, Choi JA, Chung JH, Oh JH, Lee GK, et al. (2009) Angioleiomyoma in soft tissue of extremities: MRI findings. AJR 192(6): W291-W294.

12. Jin W, Kim GY, Park SY, Chun YS, Nam DH, et al. (2010) The spectrum of vascularized superficial soft-tissue tumors on sonography with a histopathologic correlation: Part 1, benign tumors. AJR 195(2): 439-445.

13. Hwang CM, Kang BS, Hong HJ, Lee JY, SuhJH, etal. (2018) Ultrasonographic features of eccrine spiradenoma. J Ultrasound Med 37(5): 1267-1272.

14. Catalano O, Alfageme Roldan F, Solivetti FM, Scotto di Santolo M, Bouer $\mathrm{M}$, et al. (2017) Color Doppler sonography of extradigital glomus tumors. J Ultrasound Med 36(1): 231-238. 


\section{ISSN: 2574-1241}

DOI: 10.26717/BJSTR.2018.09.001857

Kazumi Fujioka. Biomed J Sci \& Tech Res

(C) This work is licensed under Creative

Submission Link: https://biomedres.us/submit-manuscript.php

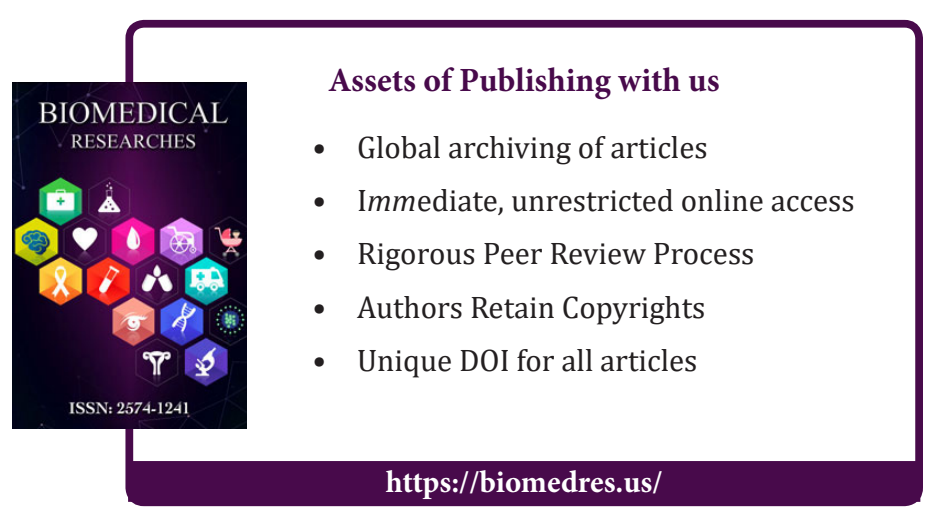

